\title{
Diffracted Fringes of Compound Nucleus Levels
}

\author{
Kazumi Ideno \\ Yabe 636-1-234, Totsuka, Yokohama, Kanagawa 244-0004, Japan
}

\begin{abstract}
We investigate a relation between the energies of nuclear excited levels and its periodic phases for nuclei with $A=14-244$ in the energy region up to several tens MeV. These levels include neutron and proton resonances, excited levels below neutron and proton separation energies and also vibrational and rotational bands in unstable nuclei. Here we use level periods less than $50 \mathrm{keV}$. We found that series of parabolic fringes appear in the plots of level energies vs. its periodic phases in various excitation modes. Distinguished fringes can be observed for neutron and proton resonances in nuclei with neutron or proton magic numbers: ${ }^{37} \mathrm{Cl},{ }^{59} \mathrm{Ni},{ }^{61} \mathrm{Ni}$ and ${ }^{62} \mathrm{Ni}$. For neutron and proton resonances in a wide mass range of nuclei, parabolic fringes with the same periods and scales can be observed at the same incident energies. Each fringe is separated by a phase difference of $1 / n$, where $n$ is an integer. We interpret the parabolic fringes as a result of interference effects based on time; a quantized phase difference of $1 / n$ can be connected to a discrete time delay of wave pulsations. These fringe spectra were compared between different nuclear excitation modes.
\end{abstract}

\section{Introduction}

A characteristic feature of compound nucleus is its complexity. For massive data of these nuclei, we tried to find out simple structure, e.g. periodic level structure for neutron resonances using a correlation function $A_{n}$ [1-3]. Many resonance levels were found to be located with periods of $\varepsilon=$ $3.06 \mathrm{eV}$ in ${ }^{180} \mathrm{Hf}\left({ }^{179} \mathrm{Hf}+n\right), \varepsilon=4.37 \mathrm{eV}$ in ${ }^{178} \mathrm{Hf}, \varepsilon=5.44 \mathrm{eV}$ in ${ }^{124} \mathrm{Sb}$ and $\varepsilon=17.6 \mathrm{eV}$ in ${ }^{169} \mathrm{Er}$. Later, Coceva et al. showed that the $4.37-\mathrm{eV}$ periodicity in ${ }^{178} \mathrm{Hf}$ is contributed mainly by $s$-wave resonances with spin $J=3$ [4]. This finding indicates that the level periodicity is relevant to symmetries such as spin. It was shown that these periodicities could not be expected from random-matrix statistical theory [5].

Detailed inspection of the $5.44-\mathrm{eV}$ periodicity in ${ }^{124} \mathrm{Sb}$ revealed that most of the resonance energies below $1200 \mathrm{eV}$ can be well approximated with integral multiples of $5.44 \mathrm{eV}$ [6]. Observation of such level sequence indicates that periodic phases of level energies play an important role in the level occurrences. This finding led to more detailed examination of a relation between level energies and its periodic phases for neutron resonances. In the analysis, we used the above periods and also ones equal to its fractions. In the plots of level energies vs. its periodic phases, there appear fringe structures, which can be approximated with parabolic curves. We can observe parabolic fringes with the same scales in many different nuclei. These fringe structures in the $\mathrm{eV}$-energy regions for medium and heavy nuclei are rather complicated. However, we found an illustrative example in a model spectrum of ${ }^{236} \mathrm{U}\left({ }^{235} \mathrm{U}+n\right)[7,8]$. When we take a period of $\varepsilon=1.09 \mathrm{eV}$, all the observed levels between 1950 and $2250 \mathrm{eV}$ are positioned on the same parabolic fringes with the same fringe centre. 
Figure 1 shows the plots of level energies vs. periodic phases for $s$-wave levels with $\operatorname{spin} J=3$ and 4 . All the more than five hundreds levels over this energy region are positioned on the parabolic fringe

(a)

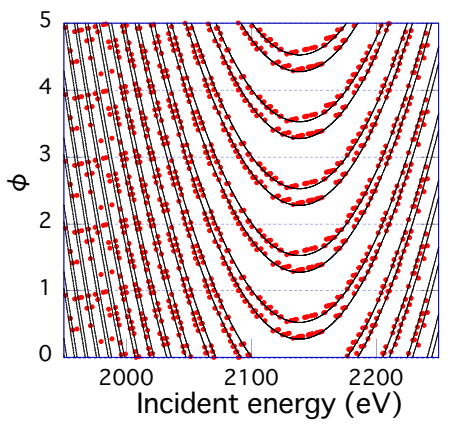

(b)

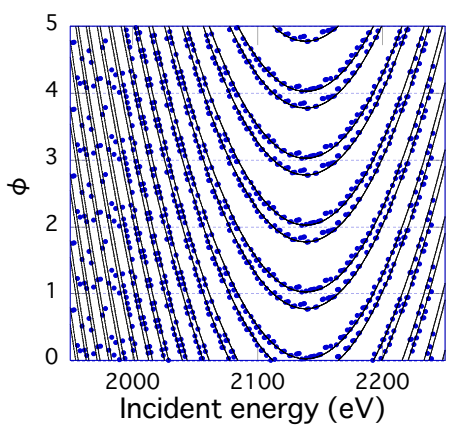

Figure 1. Plots of level energies vs. periodic phases with 1.09-eV period: $\operatorname{spin} J=3$ (a) and $J=4$ (b).

curves, which are separated from each other by a phase difference of $1 / 4$. There observed four multiple fringes between a phase difference of $\phi=1$. First and second fringe curves select $J=3$ levels, while third and fourth ones select $J=4$ levels. If we use another period of $0.437 \mathrm{eV}$, we can observe different parabolic fringes; the levels with mixed spins of $J=3$ and 4 are positioned on the same fringes where each fringe has the same fringe centre and is separated by a phase difference of 1/6. Since these level energies were obtained with a limited boundary condition [7], we call these resonance levels as a model spectrum. Later, the resonance data were revised by adopting a larger boundary condition and also by adjusting resonance parameters to fit fission cross-section measurements [9]. For the revised data [10], the fringe structure in Fig. 1 turned out to be obscured. However, the same fringes can be still traced [11].

Using nuclear data compilation [12], we performed the fringe analysis up to the energy regions of several tens $\mathrm{MeV}$. We dealt with neutron and proton resonances and nuclear excited levels below neutron and separation energies. Considering a high precision of level energies and a large number of levels, neutron resonance data play a main role in the present analysis. In favourable cases, a number of observed levels in one nucleus amounts to several thousands.

\section{Fringe analysis}

Method of fringe analysis is very simple. Let divide $i$-th level energy $E_{i}$ by a period $\varepsilon$, then we obtain

$$
E_{i}=n_{i} \varepsilon+\eta_{i}
$$

where $n_{i}$ is an integer and $\eta_{i}$ a residual of $E_{i}$. We divide both sides of Eq. 1 by $\varepsilon$. Then we obtain

$$
E_{i} / \varepsilon=n_{i}+\phi_{i}
$$

Here $\phi_{i}$ is in the range: $0 \leq \phi_{i}<1$. We define $\phi_{i}$ as a periodic phase of level energy $E_{i}$ in a periodic sequence starting from a point $E_{0}$. We usually take the starting point at zero excitation energy or zero incident energy. We treat equally such phases that differ from each other by integers: $-1,0,1,2 \ldots$ So that each level energy can have multiple numbers of phase: $\ldots \phi_{i}-1, \phi_{i}, \phi_{i}+1, \phi_{i}+2 \ldots$

We obtained diagrams of level energies vs. periodic phases using periods of $\varepsilon$. We extended the analysed energy region up to several tens $\mathrm{MeV}$. We processed nuclei with $14 \leq A \leq 244$. In choosing level periods, we took notice that low-lying excited levels in odd As isotopes [13] and ${ }^{71} \mathrm{Cu}[14]$ can 
be well approximated by integral multiples of $16.5 \mathrm{keV}$. In the following analysis, we used the period of $16.5 \mathrm{keV}$ and also its fractions such as $0.44,0.55$ and $1.65 \mathrm{keV}$.

\section{Results and discussions}

\subsection{Neutron and proton resonances}

Neutron resonance data are a promising target to perform the fringe analysis, since there observed a large number of levels with high resolution. For the present purpose, we mainly used the compiled data [12]; neutron and proton resonance levels are listed in units of excitation energy so that we can easily compare the results obtained from different excitation modes. Table 1 lists compound

Table 1. Data of typical compound nuclei, where series of parabolic fringes can be distinctively observed in the diagrams of level energies and its periodic phases. Data are from ref. [12].

\begin{tabular}{|c|c|c|c|c|}
\hline $\begin{array}{c}\text { Compound } \\
\text { nucleus }\end{array}$ & Incident particle & $\begin{array}{c}\text { Number of } \\
\text { observed levels }\end{array}$ & $\begin{array}{c}\text { Max. incident } \\
\text { energy (MeV) }\end{array}$ & $\begin{array}{c}\text { Max. excitation } \\
\text { energy (MeV) }\end{array}$ \\
\hline${ }^{37} \mathrm{Cl}$ & $n$ & 419 & 3.0 & 11.4 \\
\hline${ }^{57} \mathrm{Fe}$ & $n$ & 1242 & 9.3 & 16.9 \\
\hline${ }^{60} \mathrm{Co}$ & $n$ & 774 & 2.6 & 10.0 \\
\hline${ }^{59} \mathrm{Ni}$ & $n$ & 2437 & 9.8 & 17.6 \\
\hline${ }^{61} \mathrm{Ni}$ & $n$ & 1712 & 9.8 & 20.4 \\
\hline${ }^{62} \mathrm{Ni}$ & & & 9.8 & \\
\hline
\end{tabular}

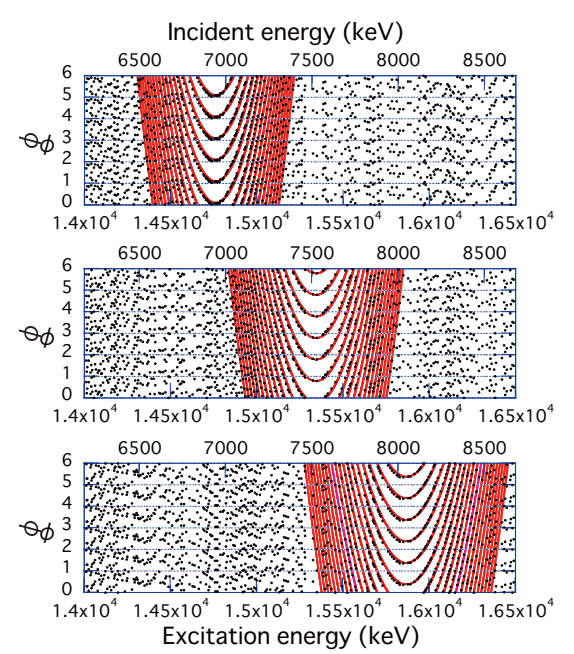

Figure 2. Plots of level energies vs. periodic phases with periods of $\varepsilon=1.32 \mathrm{keV}$ (upper), $1.49 \mathrm{keV}$ (centre) and $1.65 \mathrm{keV}$ (lower). 
nuclei, which can provide us with a starting point to understand systematically the fringe structures. Figure 2 shows the $E-\phi$ diagrams with periods of $\varepsilon=1.32,1.49$ and $1.65 \mathrm{keV}$ for neutron resonance levels in a compound nucleus ${ }^{61} \mathrm{Ni}\left({ }^{60} \mathrm{Ni}+n\right)$. It is seen that distinguished fringes appear at definite excitation energies and they are separated from each other by $\phi=1$. As the level period decreases, the centre of the fringes shifts towards a lower-excitation energy and also the scale of the parabolic curves decreases. It is noted that all the observed $\sim 220$ levels between the 14.3- and 16.5-MeV excitation energies can be covered by the parabolic fringes with level periods changing continuously from 1.32 to $1.65 \mathrm{keV}$.

Fixing a level period, for example, at $\varepsilon=1.65 \mathrm{keV}$, we examined how the parabolic fringes behave when the excitation energies or incident neutron energies decrease. Figure 3 shows plots of
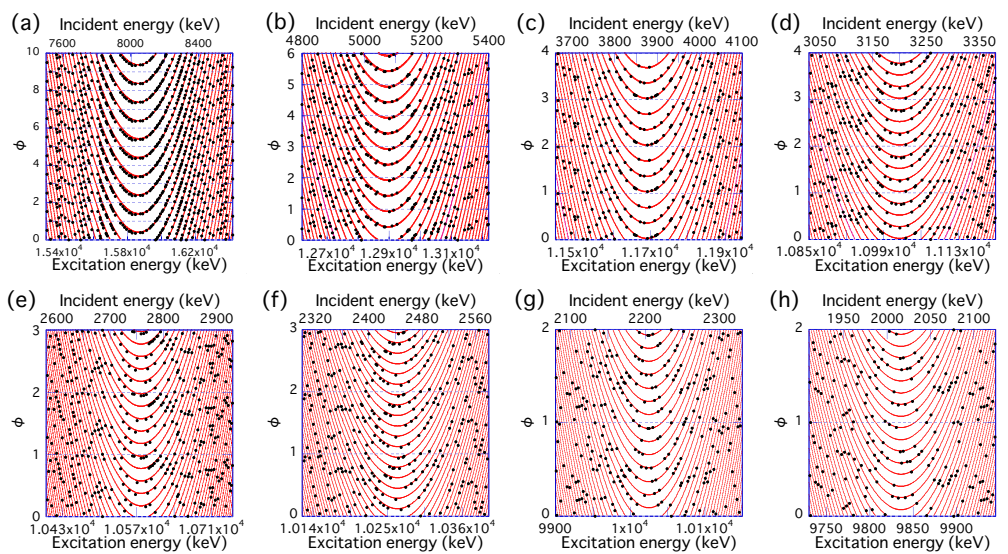

Figure 3. Plots of level energies vs. periodic phases with $\varepsilon=1.65 \mathrm{keV}$ for ${ }^{61} \mathrm{Ni}$. Multiplicity of the fringe increases continuously from $n=1$ (a) to 8 (h) as the incident neutron energy decreases. Incident neutron energies at the fringe centres are $E_{f}=8.05$ (a), 5.08 (b), 3.88 (c), 3.20 (d), 2.76 (e), 2.44 (f), 2.21 (g) and $2.02 \mathrm{MeV}(\mathrm{h})$, where $E_{f}$ denotes an incident neutron energy at a fringe centre.

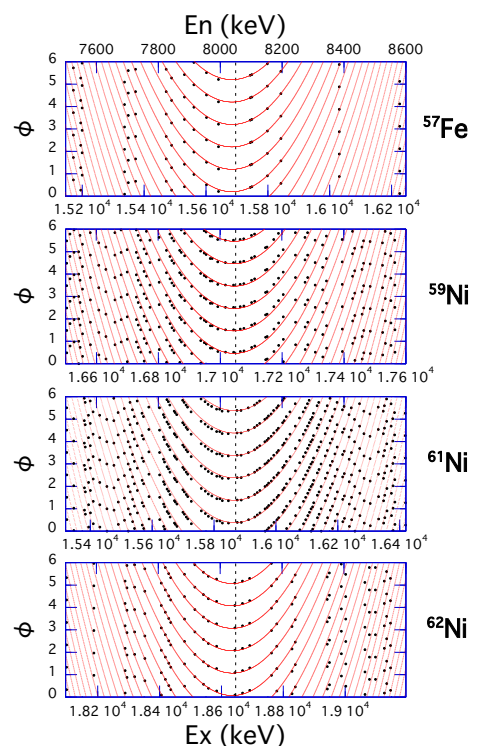

Figure 4. Plots of level energies vs. periodic phases with $\varepsilon=1.65 \mathrm{keV}$ for ${ }^{57} \mathrm{Fe},{ }^{59} \mathrm{Ni},{ }^{61} \mathrm{Ni}$ and ${ }^{62} \mathrm{Ni}$. Parabolic fringes with multiplicity of $n=1$ in these nuclei have a fringe centre at the same incident energy of $8.05 \mathrm{MeV}$. 
level energies vs. periodic phases for ${ }^{61} \mathrm{Ni}$ below an incident energy of $9.8 \mathrm{MeV}$. It is seen that at a definite excitation energy there appear parabolic fringes with a phase difference of $1 / n, n$ being an integer. Here, the phase difference of $1 / n$ means that there observed $n$ fringes between phases of $\phi$ and $\phi+1$ at the same excitation energy. We call $n$ as a multiplicity of the fringes. The multiplicity of the fringes increases from $n=1$ to 8 as the excitation energy decreases. Fringes with multiplicity larger than $n=8$ could not be resolved. Comparing observed parabolic fringes with each other among different nuclei, we found that fringe structures have a close similarity, especially among the nuclei listed in Table 1. In the nuclei ${ }^{57} \mathrm{Fe},{ }^{59} \mathrm{Ni},{ }^{61} \mathrm{Ni}$ and ${ }^{62} \mathrm{Ni}$, there observed parabolic fringes, which have fringe centres at the same incident energies and have the same scales. Figure 4 shows these parabolic fringes with multiplicity of $n=1$. Only difference among the parabolic fringes is about a bias of phase $\phi$. Figure 5 shows plots of level energies vs. periodic phases with $\varepsilon=0.55 \mathrm{keV}$ for ${ }^{60} \mathrm{Co}$, where a sequence of fringes with multiplicities of $n=1 \sim 3$ are observed below an incident energy of $1 \mathrm{MeV}$.
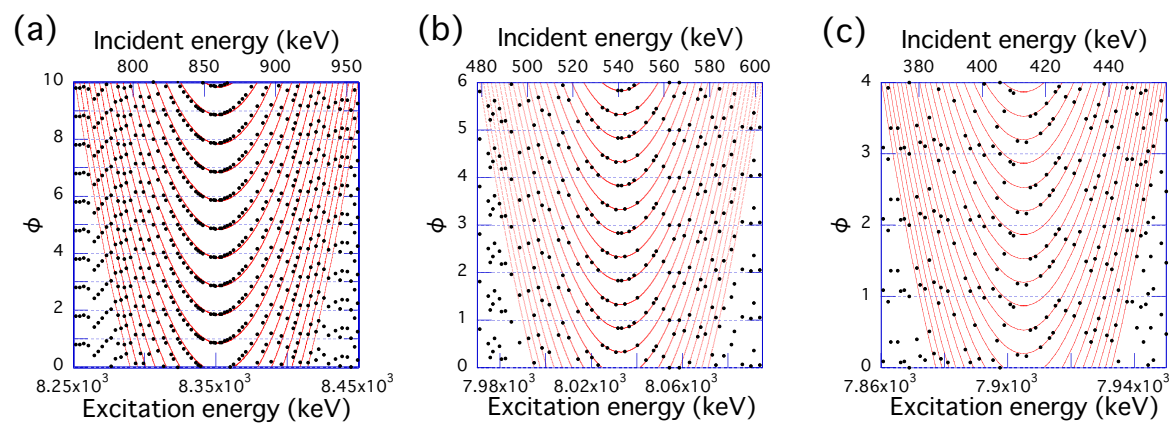

Figure 5. Plots of level energies vs. periodic phases with $\varepsilon=0.55 \mathrm{keV}$ for ${ }^{60} \mathrm{Co}$. Multiplicities of the parabolic fringes are $n=1$ (a), 2 (b) and 3 (c), while the incident energies at the fringe centres are $E_{f}=0.86$ (a), 0.54 (b) and $0.42 \mathrm{MeV}$ (c), respectively.

Figure 6 shows plots of incident energies at fringe centres vs. level periods for ${ }^{61} \mathrm{Ni}$ and ${ }^{60} \mathrm{Co}$.

(a)

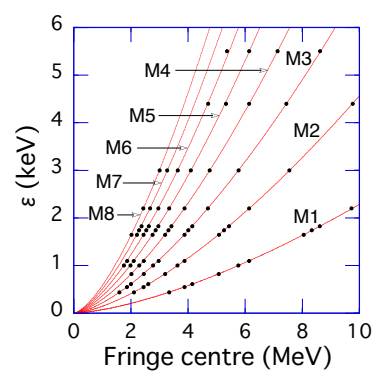

(b)

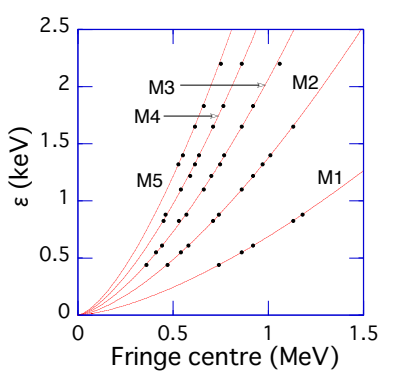

Figure 6. Plots of incident energies at fringe centres vs. level periods $\varepsilon$ for ${ }^{6 l} \mathrm{Ni}$ (a) and ${ }^{60} \mathrm{Co}$ (b). Multiplicity of parabolic fringes is indicated by $\mathrm{M} n$.

As seen from the figure, there exists a simple relation between the energies of fringe centres and level periods. The two variables can be connected with following equation to a good accuracy:

$$
\varepsilon=\alpha n E_{n} \frac{3}{2}
$$

where $\alpha$ is a constant, $n$ a multiplicity of the parabolic fringes and $E_{n}$ an incident energy at the fringe centre. Equation 3 leads to a following relation: 


$$
E_{n}: E_{1}=1: n^{\frac{2}{3}}
$$

Figure 7 shows plots of ratios $E_{n} / E_{l}$ for ${ }^{61} \mathrm{Ni}$ and ${ }^{60} \mathrm{Co}$ with level periods of $\varepsilon=0.44 \sim 5.5 \mathrm{keV}$. It is

(a)

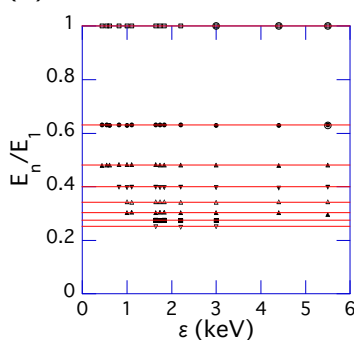

(b)

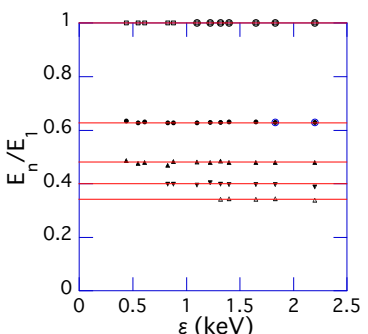

Figure 7. Plots of ratios of $E_{n}$ to $E_{l}$ for ${ }^{61} \mathrm{Ni}$ (a) and ${ }^{60} \mathrm{Co}$ (b). Multiplicities of parabolic fringes range from $n=1$ $\sim 8$ (a) and $1 \sim 5$ (b).

seen that the ratio of $E_{n}$ to $E_{l}$ is same for different level periods and applicable both to ${ }^{60} \mathrm{Co}$ and ${ }^{61} \mathrm{Ni}$. Table 2 lists the values of $E_{n} / E_{l}$ obtained from experiments and the ones predicted from formula 4 .

Table 2. Ratios of $E_{n}$ to $E_{l}$ for ${ }^{60} \mathrm{Co}$ and ${ }^{61} \mathrm{Ni} ; n$ denotes multiplicity of the parabolic fringes.

\begin{tabular}{|c|c|c|c|}
\hline $\boldsymbol{n}$ & ${ }^{\mathbf{6 0}} \mathbf{C o}$ & ${ }^{\mathbf{6 1}} \mathbf{N i}$ & $\begin{array}{c}\mathbf{1}_{\boldsymbol{n}} \boldsymbol{n}^{\mathbf{2}} \\
\text { (Predicted) }\end{array}$ \\
\hline 1 & $\begin{array}{c}\boldsymbol{E}_{\boldsymbol{n}} / \boldsymbol{E}_{\mathbf{1}} \\
\mathbf{( E x p . )}\end{array}$ & $\begin{array}{c}\boldsymbol{E}_{\boldsymbol{n}} / \boldsymbol{E}_{\mathbf{1}} \\
(\mathbf{E x p} .)\end{array}$ & 1.000 \\
\hline 2 & 1.000 & 1.000 & 0.630 \\
\hline 3 & 0.630 & 0.631 & 0.481 \\
\hline 4 & 0.480 & 0.482 & 0.397 \\
\hline 5 & 0.397 & 0.398 & 0.342 \\
\hline 6 & 0.343 & 0.343 & 0.303 \\
\hline 7 & & 0.303 & 0.273 \\
\hline 8 & & 0.274 & 0.250 \\
\hline
\end{tabular}

The predicted values agree well with ones deduced from data. This fact indicates that we can use Eq. 4 to search for the locations of parabolic fringes with different multiplicities in the same sequence. Another important parameter characterizing the parabolic fringes is a scale factor of parabolic curves. This scale factor determines the energy range around the fringe centre where these fringes dominate. Periodic phases around the fringe centre can be expressed by parabolic curves as a function of level energy:

$$
\begin{gathered}
\phi_{i j}=k_{n}\left(E-E_{n}\right)^{2}+\delta+i+\frac{j-1}{n} \\
0 \leq \delta<1 ; i=\ldots-1,0,1,2,3, \ldots ; j=1,2, \ldots, n,
\end{gathered}
$$

where $n$ is a multiplicity of the fringes, $\phi_{i j}$ denotes periodic phase at a level energy of $E, k_{n}$ a scale factor of the parabolas, $E$ a level energy, $E_{n}$ a level energy at the fringe centre and $\delta$ a constant. The constant $\delta$ corresponds to phase $\phi_{00}$ at $E=E_{n}$ with $i=0$ and $j=1$. The scale factor $k_{n}$ can have positive or minus sign. Here we deal mainly with scale factors $k_{n}$ with positive sign. All these fringes are 
parabolas of bottom-down type as shown in Figs. $1 \sim 4$. We found that a reciprocal of a scale factor $k_{n}$ is proportional to an incident energy $E_{n}$ at the fringe centre in the sequence of parabolic fringes:

$$
1 / k_{n}=\beta E_{n},
$$

where $\beta$ is a constant. Figure 6 shows plots of $1 / k_{n}$ vs. $E_{n}$ for ${ }^{61} \mathrm{Ni}$ and ${ }^{60} \mathrm{Co}$. It is seen that experimental

(a)

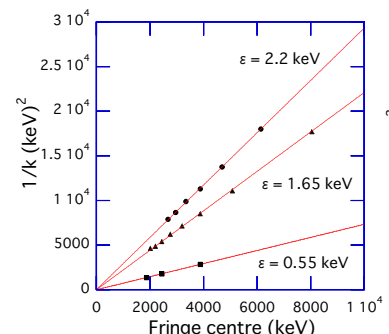

(b)

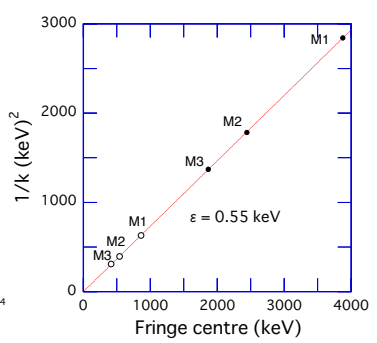

Figure 8. Plots of $E_{n}$ vs. $1 / k_{n}$ for ${ }^{61} \mathrm{Ni}$ (a) and ${ }^{60} \mathrm{Co}$ (b). (a): $n=1 \sim 3(\varepsilon=0.55 \mathrm{keV}), n=1 \sim 8(\varepsilon=1.65 \mathrm{keV})$ and $n=2 \sim 7(\varepsilon=2.2 \mathrm{keV})$; (b): upper three points (filled circles) with $n=1 \sim 3\left({ }^{61} \mathrm{Ni}\right)$ and lower three points (open circles) with $n=1 \sim 3\left({ }^{60} \mathrm{Co}\right)$.

data are on the linearly fitted lines with a good accuracy. A remarkable point in Fig. 8 (b) is that the same linear relation is commonly applicable to the sequences of parabolic fringes in ${ }^{61} \mathrm{Ni}$ and ${ }^{60} \mathrm{Co}$.

We define a fringe radius $r$ as a distance from a fringe centre at which periodic phase increases by one unit:

$$
\phi_{00}(r)=\phi_{00}(0)+1
$$

For the fringes with multiplicity of $n$, we denote the fringe radius by $r_{n}$. From Eq. 5 , fringe radius $r_{n}$ is connected with a scale factor $k_{n}$ as follows:

$$
r_{n}=1 / k_{n}^{\frac{1}{2}}
$$

Considering Eqs. 4, 6 and 8, we obtain following relation between fringe radii $r_{n}$ and $r_{1}$ belonging to the same sequence:

$$
r_{n}: r_{1}=1: n^{\frac{2}{3}}
$$

For proton resonance levels in ${ }^{37} \mathrm{Cl}\left({ }^{36} \mathrm{~S}+\mathrm{p}\right.$ ) (Table 1), we observed many parabolic fringes below an incident energy of 3.0 MeV. Plots of level energies vs. periodic phases for ${ }^{37} \mathrm{Cl}$ are shown in Fig. 9, where at an incident proton energy of $2.32 \mathrm{MeV}$, there appear distinguished parabolic fringes with multiplicities of $n=1,2$ and 3, each corresponding to level periods of $\varepsilon=0.55,1.1$ and $1.65 \mathrm{keV}$. These parabolic fringes shift towards lower energies as the level periods decrease. However, a dependence of $\varepsilon$ on $E_{n}$ is different from Eq. $3 ; \varepsilon=f n\left(E_{n}+g\right)$, where $f$ and $g$ are positive constants. This indicates that another mechanism is operated to determine the positions of these parabolic fringes. We can observe similar parabolic fringes in ${ }^{47} \mathrm{~V}$ at the same incident energy of $E_{l}=2.32 \mathrm{MeV}$ with $\varepsilon=$ $0.55 \mathrm{keV}$.

We have described mainly parabolic fringes, which have sequence structure as shown in Figs. 3, 5. We can observe parabolic fringes over a wide mass range of nuclei. Many parabolic fringes have the same incident energies at the fringe centres and the same fringe radii. Here, an exception is a phase 

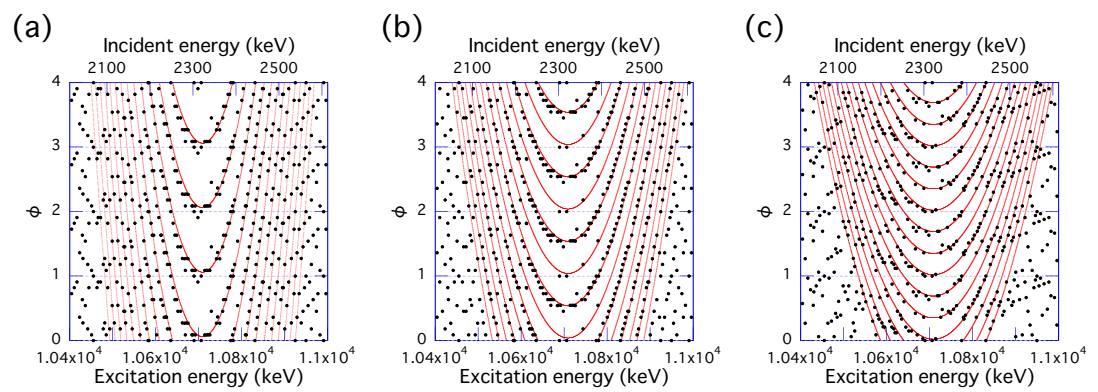

Figure 9. Plots of level energies vs. periodic phases with $\varepsilon=0.55$ (a), 1.1 (b) and $1.65 \mathrm{keV}$ (c) for ${ }^{37} \mathrm{Cl}$. Observed parabolic fringes at the incident energy of $2.32 \mathrm{MeV}$ have multiplicities of $n=1$ (a), 2 (b) and 3 (c).

constant $\delta$, which may differ from case to case. For neutron resonance levels in ${ }^{52} \mathrm{~V}$, we can observe a fringe sequence similar to the one in ${ }^{61} \mathrm{Ni}$. Also, among nuclei such as ${ }^{37} \mathrm{Cl},{ }^{41} \mathrm{Ca},{ }^{91,92} \mathrm{Zr}$ and ${ }^{208,209} \mathrm{~Pb}$, we can identify fringe sequences similar to the one in ${ }^{60} \mathrm{Co}$.

\subsection{Nuclear excited levels below neutron and proton separation energies}

We applied the fringe analysis to nuclear excited levels below neutron and proton separation energies. We searched for the same fringes as observed in neutron resonance levels. Here, we correspond nuclear excitation energies $E_{\text {exc }}$ to incident neutron energies $E_{\text {inc }}$. Figure 10 shows plots of $E_{\text {exc }}$ vs. $\phi$ with $\varepsilon=1.65 \mathrm{keV}$ for ${ }^{37} \mathrm{Cl},{ }^{61} \mathrm{Ni}$ and ${ }^{62} \mathrm{Ni}$. There appear fringes with multiplicity of $n=1$, which are

(a)

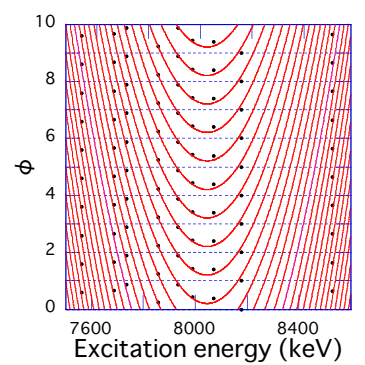

(b)

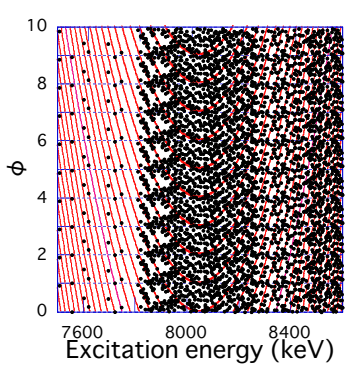

(c)

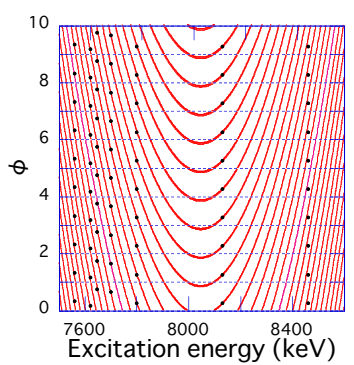

Figure 10. Plots of excitation energies vs. periodic phases for ${ }^{37} \mathrm{Cl}(\mathrm{a}),{ }^{61} \mathrm{Ni}(\mathrm{b})$ and ${ }^{62} \mathrm{Ni}(\mathrm{c})$. Neutron separation energy of ${ }^{61} \mathrm{Ni}$ is $7.82 \mathrm{MeV}$.

well reproduced by parabolic curves obtained from the $8.05-\mathrm{MeV}$ fringes in ${ }^{61} \mathrm{Ni}$. In Fig. 10 (b), dense fringes appear above $E_{\text {exc }}=7.82 \mathrm{MeV}$, which corresponds to a neutron separation energy of ${ }^{61} \mathrm{Ni}$. Fringes at lower energies can be identified also by using the same parameters $k_{n}$ and $E_{n}$ determined from ${ }^{61} \mathrm{Ni}$. The $8.05-\mathrm{MeV}$ fringes can be observed in other nuclei such as ${ }^{29} \mathrm{Si},{ }^{36} \mathrm{Cl},{ }^{41} \mathrm{~K}$ and ${ }^{55} \mathrm{Fe}$.

We surveyed fringe spectra with the level period of $16.5 \mathrm{keV}$. Parabolic fringes can be resolved for nuclei with $N \geq 14$. These fringes have a fringe radius of $r_{n}=0.5 \sim 1.5 \mathrm{MeV}$ and its fringe spectra are complex. However, there are cases where fringe spectra resemble each other in different nuclei, for example, in ${ }^{46} \mathrm{~V}$ and ${ }^{50} \mathrm{Mn}$.

\subsection{Origin of parabolic fringes in the $E$ - $\phi$ diagram}

We interpret parabolic fringes in the $E$ - $\phi$ diagram as a result of interference effects. We assume that periodic phase at $E$ around a fringe centre $E_{n}$ is a sum of periodic phases contributed from all points 
between $E$ and $E_{n}$ as a function of distance $\mathrm{x}$ from $E_{n}$ as $x / \varepsilon$; finally, periodic phase at distance $x$ from $E_{n}$ is expressed by

$$
\phi=e b \int_{0}^{x} \frac{x}{\varepsilon} \frac{d x}{\varepsilon}=e b \frac{x^{2}}{2 \varepsilon^{2}}
$$

where $e$ takes +1 in case of upward parabola and -1 in case of downward parabola; $b$ is a constant determining a scale of the fringes. Phase at $E$ is given by replacing $x$ in Eq. 10 by $\left|E-E_{n}\right|$. Parabolic fringes with multiplicity of $n$ are separated in phase by $\Delta \phi=1 / n$ from each other. If we connect a level period $\varepsilon$ with a repetition cycle of $t=2 \pi \hbar / \varepsilon$, which is called Poincaré cycle [15], time delay of $2 \pi(m-$ $1) \hbar / n \varepsilon$ can correspond to $m$-th fringe; here, $m=1,2, \ldots, n$. Time-based treatment of nuclear reaction is also made [16].

Downward parabolas can be actually observed in fringe spectra, but its role is not clear compared to upward parabolic fringes. Empirical rules on the correlation between scale factors and positions of parabolic fringes have been determined for typical examples, but we are far from understanding the whole picture of fringe structure.

\section{Conclusion}

Parabolic fringes with the same scale factors are observed at the same incident neutron energies below $10 \mathrm{MeV}$ in nuclei such as ${ }^{55} \mathrm{Fe},{ }^{57} \mathrm{Fe},{ }^{59} \mathrm{Ni},{ }^{61} \mathrm{Ni}$ and ${ }^{62} \mathrm{Ni}$. Spin assignments of these levels are limited to the energy region below $1 \mathrm{MeV}$. To study symmetry dependences of parabolic fringes, we need spin information on these levels at higher energies.

We interpret the parabolic fringes as a result of interference effects on periodic phases; a quantized phase difference of $1 / n$ can be connected with a discrete time delay of wave pulsations caused by incident particles.

\section{References}

1. S. I. Sukhoruchkin, Sov. J. Nucl. Phys. 10, 285 (1970)

2. K. Ideno and M. Ohkubo, J. Phys. Soc. Jpn. 30, 620 (1971)

3. K. Ideno, J. Phys. Soc. Jpn., 37, 581 (1974)

4. C. Coceva et al., Proc. Int. Conf. on Statistical Properties of Nuclei, J. B. Garg, 447, Plenum, N.Y., 1972

5. K. Ideno, Proc. Int. Conf. on Nuclear Data for Science and Technology, 783, Mito, 1988

6. K. Ideno, Proc. Int. Conf. on Neutrons in Research and Industry, SPIE, Washington, vol. 2867, 398, 1997

7. L. C. Leal et al., Nucl. Sci. Eng. 109, 1 (1991)

8. Landolt-Boernstein, New Series vols. 16/B, Springer, Heidelberg, 1998

9. L. C. Leal et al., Nucl. Sci. Eng. 131, 220 (1999)

10. Landolt-Boernstein, New Series vols. 16/C, Springer, Heidelberg, 2004

11. K. Ideno, AIP915, 819 (2008)

12. Landolt-Boernstein, New Series vols. I/25, A, B, Springer, 2012; I/25C, D, E, 2013

13. I. Hossain et al., Phys. Rev. C 58, 1318 (1998)

14. T. Ishii et al., Phys. Rev. Lett., 84, 39 (2000)

15. K. Izumo, Prog. Theor. Phys. 54, 1378 (1975)

16. M. Ohkubo, Phys. Rev. C53, 1325 (1996); ibid., C73, 054609 (2006); C87, 014608 (2013) 